\title{
Turbulent Origin of the Galactic-Center Magnetic Field: Nonthermal Radio Filaments
}

\author{
Stanislav Boldyrev ${ }^{1}$ and Farhad Yusef-Zadeh ${ }^{2}$
}

\begin{abstract}
A great deal of study has been carried out over the last twenty years on the origin of the magnetic activity in the Galactic center. One of the most popular hypotheses assumes milli-Gauss magnetic field with poloidal geometry, pervading the inner few hundred parsecs of the Galactic-center region. However, there is a growing observational evidence for the large-scale distribution of a much weaker field of $B \lesssim 10 \mu G$ in this region. Here, we propose that the Galactic-center magnetic field originates from turbulent activity that is known to be extreme in the central hundred parsecs. In this picture the spatial distribution of the magnetic field energy is highly intermittent, and the regions of strong field have filamentary structures. We propose that the observed nonthermal radio filaments appear in (or, possibly, may be identified with) such strongly magnetized regions. At the same time, the large-scale diffuse magnetic field is weak. Both results of our model can explain the magnetic field measurements of the the Galactic-center region. In addition, we discuss the role of ionized outflow from stellar clusters in producing the long magnetized filaments perpendicular to the Galactic plane.
\end{abstract}

Subject headings: Galaxy: center — ISM: general — MHD — turbulence

\section{Introduction.}

Magnetic field is ubiquitously inferred in the interstellar medium. Although its origin and large-scale (Galactic-scale) structure is still debated, it is suggested by observations that the Galactic field strength is of the order of few $\mu G$ (e.g., Zweibel \& Heiles 1997). Moreover, the fluctuating part of the field is of the order of its large-scale component, and that the field is roughly in equipartition with the gas pressure and the cosmic-ray pressure.

\footnotetext{
${ }^{1}$ Department of Astronomy and Astrophysics, University of Chicago, 5640 S. Ellis Ave., Chicago, IL 60637, boldyrev@uchicago.edu

${ }^{2}$ Department of Physics and Astronomy, Northwestern University, Evanston, IL 60208, zadeh@northwestern.edu
} 
Less consensus has been reached about the origin, structure, and strength of the magnetic field in the center of the Galaxy (central hundreds of parsecs). The earliest study of large-scale magnetic activity in this region dates back to the discovery of nonthermal radio filaments more than 20 years ago (Yusef-Zadeh, Morris \& Chance 1984). These filaments are fairly straight, about ten to hundred parsec long and about a fraction of a parsec wide. The linear polarization of these filaments indicates that they radiate due to synchrotron emission of energetic, non-thermal particles following the magnetic field lines (the origin of these particles being a separate issue). The rigidity and the linearity of the filaments distributed within the zone of molecular gas having high density and velocity dispersion were used to make an estimate of the magnetic field. This dynamical argument estimated a milli-Gauss field (Yusef-Zadeh \& Morris 1987; Serabyn \& Güsten 1991; Morris \& Serabyn 1996; Bicknell \& Li 2001). In the intervening years, a number of additional filaments have been discovered (see the review by Yusef-Zadeh 2003; Lang et. al. 1999; Reich 2003; Nord et. al. 2004; LaRosa et al. 2004; Yusef-Zadeh, Hewitt \& Cotton 2004).

In early works, nonthermal radio filaments were detected predominantly perpendicular to the Galactic plane. This prompted the theories suggesting that strong magnetic field, of the order of milli-Gauss, is uniformly pervasive in the Galactic center, and relativistic electrons are injected into this pre-existing field at some places thus "lighting up" the corresponding magnetic-field lines. One of such theories assumed that the magnetic flux was already strong enough in the protogalaxy, and then it was confined to the Galactic disk during the Galactic disk formation. The subsequent matter inflow into the center further concentrated the poloidal flux (see e.g., Sofue \& Fujimoto 1987; Benford 1988; Morris 1994; Chandran, Cowley \& Morris 2000). Alternatively, certain local mechanisms have been proposed to explain the production of non-thermal radio filaments (see e.g., Rosner \& Bodo 1996; Shore \& LaRosa 1999; Yusef-Zadeh 2003). In one model, the magnetic field was amplified by thermal instability (Rosner \& Bodo 1996), whereas in another the filaments were considered to be magnetized wakes produced as a result of an interaction of the molecular clouds with a Galactic center wind (Chevalier 1992; Shore \& LaRosa 1999).

The conventional theory that assumes milli-Gauss magnetic field with poloidal geometry, surrounding the Galactic center, needs to explain not only the origin of $\sim 4 \times 10^{54}$ ergs of energy trapped in the magnetic field within a $100 \mathrm{pc}$ radius, but also the mechanism of anchoring of the strong magnetic field lines. Moreover, this theory has recently encountered a number of serious observational challenges. First, more than eighty radio filaments have already been detected, and their directions do not always run perpendicularly to the galactic plane (e.g., Lang et. al. 1999; LaRosa et al. 2004; Nord et. al. 2004; Yusef-Zadeh, Hewitt $\&$ Cotton 2004). Second, a number of arguments based on Zeeman and Faraday-rotation measurements toward the Galactic center as well as on the estimates of synchrotron life- 
time, suggest that the pervasive magnetic field strength should not exceed $100 \mu \mathrm{G}$ (Uchida and Güsten 1995; Yusef-Zadeh 2003; LaRosa et. al. 2005). Third, the anisotropic property of the scattering medium, which is thought to be seated in the Galactic center region, shows a random orientation of scatter-broadened $\mathrm{OH} / \mathrm{IR}$ stars in the central $50^{\prime}$ of the Galactic center (van Langevelde et. al. 1992; Frail, et al. 1994). The combined anisotropy of OH/IR stars, $\mathrm{OH}(1720 \mathrm{MHz})$ masers, as well as the anisotropy of the rotation measure of the nonthermal filament G359.1-0.54 (Yusef-Zadeh et. al. 1997) are inconsistent with a strong poloidal space-filling magnetic field pervading the Galactic-center region. Fourth, the recent discovery of X-ray emission from three nonthermal filaments ( $\mathrm{Lu}$ et. al. 2003; Sakano et. al. 2003) has suggested that X-ray emission from G359.90-0.06 could be produced by inverse Compton scattering (ICS) of far-infrared photons from dust by the relativistic electrons responsible for the radio synchrotron emission (Yusef-Zadeh et. al. 2005). The production of X-ray emission from ICS allows an estimate of the magnetic field strength of order $0.1 \mathrm{mG}$ within the nonthermal filament. Fifth, sub-millimeter polarization measurements of dust emission from several Galactic-center molecular clouds show that the large-scale distribution of the magnetic field follows a toroidal geometry along the Galactic plane (Novak et al. 2000; Chuss et al. 2003). And last, the recent discovery of diffuse nonthermal structure in the Galactic center by LaRosa et. al. (2005) imposes even stronger limitation of the largescale magnetic field strength, suggesting that the pervasive magnetic field in the Galactic center is rather weak, of order $10 \mu G$. This is consistent with earlier measurements of rotation measure distribution of extragalactic radio sources projected toward the Galactic center (Roy 2004), and with the observations of the nonthermal filament G359.1-0.2 (Gray et al. 1995), indicating magnetic field strength of the Faraday screen, ranging between 1 and $10 \mu \mathrm{G}$.

In other words, several observations indicate that although the magnetic field in the nonthermal filaments may be strong, it is not representative of the large-scale pervasive magnetic field in the Galactic center. A theory of the Galactic center is thus in demand, which would provide a robust explanation for both weak pervasive magnetic field and local, randomly oriented, strongly magnetized filamentary structures. In the present paper we attempt to propose such a unifying explanation. We suggest that, quite generally, magnetic structure of the Galactic center can be derived from its strong turbulent activity. We argue that the pervasive magnetic flux in the Galactic center is quite weak, however, the magnetic field is significantly amplified locally so that the regions of strong field have filamentary structures, as an inevitable consequence of magnetic dynamo mechanism associated with such a turbulent activity. We then propose that the nonthermal radio filaments are observed in (and possibly may be identified with) such strongly magnetized regions. A physical picture of strongly turbulent Galactic center is motivated by radio observations of compact sources indicating heavily scatter-broadened radio sources toward the inner degree of the Galactic center (van 
Langevelde et. al. 1992). The most extreme scattering medium in the Galaxy is argued to be located within the inner few hundred parsecs of the Galactic center (Yusef-Zadeh et al. 1994; Lazio \& Cordes 1998). This scattering medium is considered to be denser than in the disk, $n_{e} \sim 1 \mathrm{~cm}^{-3}$, by one to two orders of magnitude (Lazio \& Cordes 1998).

\section{Inhomogeneous MHD Turbulence}

Magnetohydrodynamic turbulence is generally investigated, both analytically and numerically, for idealized homogeneous and isotropic settings. However, astrophysical turbulence is almost always inhomogeneous and anisotropic. In the situation when the outer scale of turbulence (the correlation scale of turbulent fluctuations) is much smaller than the scale of turbulent intensity variation, the approximation of homogeneity works well. However, as we show in the next section, in the Galactic center these two scale lengths are of the same order. In this case, the effect of magnetic-field amplification by turbulent motion should be considered in conjunction with another effect - magnetic-field expulsion from the region of stronger turbulence. In the present section we propose a description of magnetic-field structure in inhomogeneous turbulence.

As first noted by Batchelor (1950), when fluid viscosity and resistivity are negligible, and if the magnetic field acting back on the fluid is weak, the evolution equation for magnetic field, $\partial_{t} \mathbf{B}=\nabla \times[\mathbf{v} \times \mathbf{B}]$, formally coincides with the equation for the vorticity, $\omega=\nabla \times \mathbf{v}$. When a weak diffuse magnetic field is amplified by turbulence, it is randomly stretched and folded so that magnetic energy distribution becomes spatially intermittent. Analogously to a distribution of vorticity in hydrodynamic turbulence (e.g., She, Jackson \& Orszag 1990; Frisch 1995; Kaneda et. al. 2003), the strongest field is concentrated in filamentary structures, where it reaches the equipartition with the turbulent energy (e.g., Nordlund et. al. 1992). The characteristic rate of field amplification is the turbulent-eddy turnover rate, and the characteristic length of the filamentary structures is the outer-scale of turbulence.

If the magnetic field were confined to the turbulent region (as, for example, in most numerical simulations), the number of filamentary structures would increase until the magnetic field would become strong everywhere (e.g., Cattaneo 1996). If, however, turbulence is confined to some spatial region but magnetic field is not, then the so-called diamagnetic effect comes into play. Namely, quite generally, large-scale magnetic flux tends to be expelled from the turbulent region. In other worlds, when the intensity of fluid turbulence varies in space, the large-scale magnetic field is transported in the direction of weaker turbulence (Zeldovich 1956; Parker 1975; Vainshtein \& Kichatinov 1983; Landau, Lifshitz \& Pitaevskii 1995). The characteristic time for flux expulsion is the turbulent diffusion time. Denote $l_{0}$ the largest 
scale of turbulence, and $v_{0}$ the eddy turnover velocity at this scale, then this time is estimated as $\tau_{d} \sim L^{2} / \eta_{T}$, where $L$ is the size of the turbulent region (we assume $l_{0}<L$ ) and $\eta_{T}=l_{0} v_{0}$ is turbulent diffusivity. As we discuss in the next section, the size of the turbulent region in the Galactic center is comparable with the outer scale of turbulence, or may be bigger by a factor of few, $L \gtrsim l_{0}$. We may therefore conjecture that in a steady state the magnetized filamentary structures have a chance to diffuse out of the turbulent region, so that the magnetic field does not become strong everywhere in the turbulent region. Rather, the strongest field is embedded by magnetic flux tubes.

We propose that these magnetic structures are the places where the Galactic-center nonthermal radio filaments appear. In this model relativistic particles may be pervasive (say, cosmic rays), while the regions of strong magnetic field are spatially intermittent, and the average magnetic field is weak. The synchrotron emissivity that is proportional to $B^{(1+\gamma) / 2}$, where $\gamma$ is the spectral index of the power-law energy spectrum of relativistic particles (Salter \& Brown 1988) and $B$ is the component of the magnetic field perpendicular to the line of sight, is enhanced along the filaments as the magnetic field is amplified. For example, in the case of a steep spectrum of relativistic particles measured at high frequencies, with $\gamma \sim 3$ (Yusef-Zadeh et. al. 2005; Anantharamaiah et al. 1991; Lang et. al. 1999) the synchrotron emission may increase by two orders of magnitude with respect to background emission if the magnetic field is amplified by only a factor of 10, assuming that the cosmic-ray particle density is constant. In the case of low value of $\gamma \sim 2$, as measured at low frequencies (LaRosa et. al. 2000), the synchrotron emissivity could increase only by a factor of 30. Alternatively, however, the relativistic particle density may not be constant. For example, these particles could be injected locally by compact sources that produce relativistic particles (Yusef-Zadeh \& Königl 2004). We now apply the above general arguments to estimate the parameters of the nonthermal radio filaments.

\section{Magnetic-Field Structure in the Galactic Center}

We consider that characteristic gas distribution in the Galactic center consists of four phases: cold, cool, warm, and hot (Oka et. al. 2005). The cold phase is a molecular gas whose temperature is $T_{\text {cold }} \sim 70 \mathrm{~K}$, and density is $n_{\text {cold }}>10^{4} \mathrm{~cm}^{-3}$. These molecular clouds occupy only a small fraction of space, with a filling factor $f_{\text {cold }} \lesssim 0.01$. The cool phase consists of atomic hydrogen with temperature $T_{\text {cool }} \sim 250 \mathrm{~K}$, and density $n_{\text {cool }} \sim 10^{2} \mathrm{~cm}^{-3}$. Its filling factor can be large, $f_{\text {cool }} \sim 0.5-1$. The worm phase is ionized gas, with temperature $T_{\text {warm }} \sim$ $10^{4} \mathrm{~K}$, density $n_{\text {warm }} \sim 10^{2} \mathrm{~cm}^{-3}$ and filling factor $f_{\text {warm }} \lesssim 1$ based on the scattering measure of the Galactic center sources (Lazio \& Cordes 1998). The hot phase of the Galactic center 
has been modeled to have two plasma components, the soft component with $\mathrm{kT} \sim 0.8 \mathrm{keV}$ and the hard one with a $\mathrm{kT} \sim 8 \mathrm{keV}$ (e.g., Muno et al. 2004; Koyama et al. 1996). The mean electron density of hot gas is estimated to be $n_{\text {hot }} \sim 0.3-0.4 \mathrm{~cm}^{-3}$, and the filling factor is $f_{\text {hot }} \lesssim 1$ (Koyama et al. 1996). Considering that all these phases co-exist with each other, it is difficult to argue that they are in thermal pressure equilibrium. Rather, observations of molecular and atomic line emission (e.g., Tsuboi et al. 1999; Oka et. al. 2005; Geballe et al. 2005) suggest that these phases are turbulent with turbulent velocity dispersion of order $v_{0} \sim 10-50 \mathrm{~km} / \mathrm{sec}$. We therefore believe that it is reasonable to assume that their turbulent energies rather than thermal energies are of the same order, and that strong turbulence plays an essential role in the Galactic-center gas dynamics.

To estimate the outer scale of the turbulence, we need to specify possible mechanisms of turbulence generation. We may first assume that the turbulence is stirred through supernovae explosions, and accept that the rate of explosions in the central $50 \mathrm{pc}$ is about 1 per $10^{5}$ years (Figer et al 2004; LaRosa et. al. 2005). Assuming that a supernova shell expands with velocity $v_{s h} \sim 10^{7} \mathrm{~cm} / \mathrm{s}$, we deduce that the outer scale of turbulence in the Galactic center environment is of order $l_{0} \sim 30 \mathrm{pc}$. (A shell expands uninterrupted by another supernovae explosion up to a radius $l_{0}$ during time $l_{0} / v_{s h}$. Another supernova will distort the expanding shell if the following condition is satisfied, $l_{0}^{3} /[50 \mathrm{pc}]^{3} \sim\left[v_{s h} / l_{0}\right] 10^{5} \mathrm{yrs}$, which leads to the above magnitude of $l_{0}$ ). This value provides a simple, but quite rough estimate. A more plausible consideration is that the large-scale turbulence is not uniform and isotropic. Stronger turbulence may be produced by massive star-forming activity in the Galactic center, which appears to occur predominantly in star clusters. In this picture turbulence could be driven by strong stellar winds from these young massive clusters. These winds can collide on scales characterized by the distance between mass-losing stars within a cluster or clusters themselves, $\sim 10-20$ pc along the Galactic plane. The winds could also be correlated on much larger scales $\sim 100$ pc perpendicular to the Galactic plane (where the gas density drops off), driving thermal gas away in this direction.

A number of studies have recently suggested that the high pressure environment of the Galactic center went through a mini-starburst activity ten million years ago (e.g., BlandHawthorn \& Cohen 2003). In particular, the large scale $1^{0}$ omega-shaped lobe structure (Sofue \& Handa 1984) shows the largest concentration of nonthermal filaments in the Galactic center region. This large concentration of magnetic filaments appears in the vicinity of the footprints of the large-scale lobe where the bright HII complex of Sgr C (e.g., G359.5-0.0) (Yusef-Zadeh, Hewitt \& Cotton 2004; Nord et. al. 2004) as well as the radio continuum arc (l $\sim 0.2^{0}$ ) are located. We suggest that the presence of the longest and brightest vertical nonthermal filaments is phenomenologically tied to the origin of the omega-shaped lobe structure. In this picture, the outer scale of the turbulence stretching the field lines is in the 
direction away from the plane and is identified by the past activity of the collision of the winds and supernovae. In particular, an anisotropic distribution of thermal and nonthermal gas in the direction along and away from the Galactic plane can naturally explain the fact that the longest filaments run predominantly perpendicularly to the Galactic plane. In this picture, we speculate that the outer scale of turbulence could be an order of magnitude larger away from the plane than parallel to the plane.

When strong turbulence is switched on in a highly conducting medium permeated by weak, diffuse magnetic field, the field is amplified by random fluid motion, under the dynamo mechanism (e.g., Kazantsev 1968; Moffatt 1978; Vainshtein \& Kichatinov 1983; Kulsrud \& Anderson 1992; Schekochihin, Boldyrev \& Kulsrud 2002). As we discussed in the previous section, at the initial stage of amplification, the strong-field regions have morphology of filaments (e.g., Nordlund et. al. 1992). The magnetic field inside these filamentary structures increases until the Lorentz force is strong enough to prevent further field amplification. This happens in several dynamical, eddy turn-over times, $\tau \sim l_{0} / v_{0} \sim 10^{6}-10^{7} \mathrm{yrs}$. The balance of the Lorentz force inside a filamentary structure and the surrounding turbulent forces gives the magnetic field inside the structure, $B_{f} \gtrsim 0.1 \mathrm{mG}$. The length of a filamentary structure cannot exceed the outer scale of turbulence $l_{0}$, since the strongest eddy that can stretch the structure has the scale $l_{0}$. The characteristic distance between filamentary structures is also of the order of $l_{0}$. However, shorter and weaker structures are possible; they can appear closer to each other since they are created by smaller and less energetic eddies.

Assume now, that mean magnetic field in the Galactic center is roughly of the same order as in the rest of the Galaxy, $B_{0} \lesssim 10 \mu G$. The field is amplified by turbulent motion locally. Consider the regions of space where the field is amplified by at least a factor of 10 (i.e., $B_{f}>0.1 \mathrm{mG}$ ). Such strongly magnetized filamentary structures should be encountered with a rather small probability, i.e. they should have a rather small volume filling factor. To illustrate this, one can use the probability density function (PDF) of magnetic-field strength. Since the pervasive field $B_{0}$ is weaker than the value dictated by equipartition with turbulent energy, this function can be obtained from the kinematic dynamo theory. In the well investigated homogeneous and isotropic case, this theory predicts that such a function is log-normal, $P(B) \mathrm{d} B=\sqrt{C / \pi} \exp \left[-C \log ^{2}\left(B / B_{0}\right)\right] \mathrm{d} B / B$, where $C$ is some coefficient, $C \sim$ $l_{0} /\left(v_{0} t\right)$. This prediction is in good agreement with numerical simulations, (e.g., Cattaneo 1996; Schekochihin et. al. 2005). If the field-amplification time is approximately the eddy turnover time, then $C \sim 1$ (this crude estimate suffices for our illustrative purposes). Using this formula one can estimate that the probability (or, the volume filling factor) of filaments with $B>10 B_{0}$ is quite small, $f \sim 10^{-4}$. Assuming that the number of the strongest filaments per outer scale of turbulence is of order one, and the length of such filaments is $l_{0}$, we estimate $l_{0} l_{f}^{2} \sim f l_{0}^{3}$. We then find the width of the filaments as $l_{f}<0.01 l_{0}$. This rough 
estimate is in agreement with observations (Yusef-Zadeh, Hewitt \& Cotton 2004).

Interestingly, the theory of MHD turbulence may also allow us to explain another peculiar and puzzling property of nonthermal radio filaments. Quite often, these filaments are observed to be split into multiple filaments running parallel to each other. In fact, there are more such multiplets than single isolated filaments (see, e.g., Lang et. al. 1999; Yusef-Zadeh, Hewitt \& Cotton 2004). We may speculate that such multiple filamentary structure could be identified with folded structure of magnetic field inside strongly magnetized regions, as is consistently observed in numerics (see, e.g., Cattaneo 1996; Schekochihin et. al. 2005).

In conclusion, we have proposed a new interpretation of the magnetic-field structure in the Galactic-center region where numerous unique nonthermal filaments have been detected. The main idea of our approach is that the magnetic-field distribution in the Galactic-center environment is inherently and universally related to the strong turbulent activity observed in this region. Such turbulence locally amplifies the diffuse background magnetic field and concentrates the strong field in thin and elongated, randomly oriented filamentary structures. The distribution of the longest filamentary structures can be anisotropic, however, due to anisotropic character of the large-scale turbulence driven by collisions of stellar winds. The nonthermal radio filaments originate inside such structures, or can possibly be identified with these structures themselves. The proposed turbulent origin of nonthermal filaments in the Galactic center distinguishes our approach from other theories in that our model is mainly driven by observations, it is based on quite general results of the theory of turbulence, and it resorts to minimal non-standard assumptions.

We are grateful to Samuel Vainshtein and Ellen Zweibel for useful discussions. The work of S.B. was supported by the NSF Center for Magnetic Self-Organization in Laboratory and Astrophysical Plasmas at the University of Chicago.

\section{REFERENCES}

Anantharamaiah, K.R. et al. 1991, MNRAS, 249, 262.

Batchelor, G. K., 1950, Proc. Royal. Soc. London, A 201, 405.

Benford, G., 1988, ApJ, 333, 735.

Biskamp, D., 2003, Magnetohydrodynamic Turbulence. (Cambridge University Press, Cambridge.).

Biskamp D., \& Müller W.-C., 2000 , Phys. Plasmas, 7, 4889-4900. 
Bicknell, G. V. \& Li, J., 2001, ApJ, 548, L69.

Bland-Hawthorn, J. \& Cohen, M., 2003, ApJ, 582, 246.

Cattaneo, F., 1996, in SCORe'96: Solar Convection and Oscillations and Their Relationship (Kluwer Academic Publishers, Netherlands 1997, eds. F. P. Pijpers et. al.), 201-222.

Chandran, B.D.G., Cowley, S.C. \& Morris, M., 2000, ApJ, 528, 723.

Chevalier, R., 1992, ApJ, 397, L39.

Chuss, D.T., Davidson, J.A., Dotson, J.L., Dowell, C.D., Hildebrand, R.H. et al., 2003, ApJ, $599,1116$.

Figer, D. F., Rich, R. M., Kim, S. S., Morris, M., \& Serabyn, E., 2004, ApJ, 601, 319.

Frail, D.A., Diamond, P.J., Cordes, J.M., \& van Langevelde, H.J., 1994, ApJ, 427, L43.

Frisch, U., 1995, Turbulence. (Cambridge University Press).

Geballe, T.R., Oka, T., Goto, M., Usuda, T., McCall, B.J. 2005, AAS, 206, 3701.

Gray, A.D., Nicholls, J., Ekers, R.D. \& Cram, L.E., 1995, ApJ, 448, 164.

Kaneda, Y., Ishihara, T., Yokokawa, M., \& Itakura, K., Uno, A., 2003, Phys. Fluids. 15, L21.

Kazantsev, A. P., 1968, Soviet Phys.-JETP, 26, 1031.

Koyama, K., Maeda, Y., Sonobe, T., Takeshima, T., Tanka, Y. \& Yamauchi, S., 1996, PASJ, 48, 249.

Kulsrud, R. M., 2005, Plasma Physics for Asrophysics. (Princeton University Press).

Kulsrud, R. M. \& Anderson, S. W., 1992, ApJ, 396, 606.

Landau, L. D., Lifshitz, E. M. \& Pitaevskii, L. P., 1995, Electrodynamics of Continuous Media (Butterworth-Heinemann).

Lang, C., Anantharamaiah, K. R., Kassim, N. E., \& Lazio, T. J. W., 1999, ApJ, 521, L41.

LaRosa, T. N., Brogan, C. L., Shore, S. N., Lazio, T. J. W., Kassim, N. E., \& Nord, M. E., 2005, ApJ, in press

LaRosa, T. N., Kassim, T.N., Lazio, T.J.W. \& Hyman, S.T. 2000, AJ, 119, 207. 
LaRosa, T. N., Nord, M.E., Lazio, T. J. W., \& Kassim, N. E., 2004, ApJ, 607, 302.

Lazio, T.J.W. \& Cordes, J., 1998, ApJ, 505, 715.

Lu, F.G., Wang, Q.D., \& Lang, C.C., 2003, AJ, 126, 319-326.

Moffatt, H. K., 1978, Magnetic Field Generation in Electrically Conducting Fluid (Cambridge University Press, Cambridge).

Morris, M., 1994, (in Genzel R \& Harris, A. I., eds. Nuclei of Normal Galaxies: Lessons from the Galactic Center, NATO ASI Ser. C. Dordrecht: Kluwer, p. 185.)

Morris, M \& Serabyn, E., 1996, ARA\&A, 34, 645.

Muno, M. P., Baganoff, F. K., Bautz, M. W. , Feigelson, E. D., Garmire, G. P., Morris, M. R., Park, S., Ricker, G. R., \& Townsley, L. K., 2004, ApJ, 613:326-342.

Novak, G., Dotson, J.L., Dowell, C.D., Hildebrand, R.H., Renberger, T. \& Schleuning, D.A., 2000, ApJ, 529, 241.

Nord, M.E., Lazio, T.J.W., Kassim, N.E., Hyman, S.J., LaRosa, T.N., Brogan, C.L. \& Duric, N., 2004, AJ, 128, 1646.

Nordlund, A., Brandenburg, A., Jennings, R. L., Rieutord, M., Ruokolainen, J., Stein, R. F., \& Tuominen, I., 1992, ApJ, 392, 647.

Oka, T., Geballe, T. R., Goto, M., Usuda, T. \& McCall, B. J., 2005, ApJ, to appear; (astro-ph/0507463)

Parker, E. N., 1975, ApJ, 202, 523.

Reich, W., 2003, A\&A, 401, 1023.

Rosner, R. \& Bodo, G., 1996, ApJ, 470, L49.

Roy, S., 2004, Bull. Astr. Soc. India (arXiv:astro-ph/0411687)

Sakano, M., Warwick, R.S., Decourchelle, A. \& Predhel, P., 2003, MNRAS, 340, 747-751.

Salter, G. \& Brown, R.L. 1988, in "Galactic and Extragalactic Radio Astronomy", eds: G.L. Verschuur \& K.I. Kellermann, Publisher: Springler-Verlag.

Schekochihin, A., Boldyrev, S., \& Kulsrud, R., 2002, ApJ, 567, 828; astro-ph/0103333. 
Schekochihin, A. A., Cowley, S. C., Taylor, S. F., Maron, J. L., \& McWilliams, J. C., 2004, ApJ, 612, 276-307.

Serabyn, E \& Güsten, R., 1991, Astron. Astrophys., 242, 736.

She, Z. S., Jackson, E., \& Orszag, S. A., 1990, Nature, 344, 226-228.

Shore, S.N. \& LaRosa, T.N., 1999, ApJ, 521, 587.

Sofue, Y. \& Handa, T. 1984, Nature, 310, 568

Sofue, Y. \& Fujimoto, M., 1987, PASJ, 39, 843.

Tsuboi, M., Handa, T. \& Ukita, N., 1999, ApJS, 120, 1.

Uchida, K.I. \& Güsten, R., 1995, A\&A, 298, 473.

van Langevelde, H.J., Frail, D.A., Cordes, J.M. \& Diamond, P.J., 1992, ApJ, 396, 686.

Vainshtein S. I. \& Kichatinov, L. L., 1983, Geophys. Astrophys. Fluid Dynamics, 24, 273.

Yusef-Zadeh, F., Wardle, M., Muno, M., Law, C. \& Pound, M. 2005, Advances in Space Research, 35, 1074.

Yusef-Zadeh, F., 2003, ApJ, 598, 325.

Yusef-Zadeh, F. \& Königl, A., 2004, in Proceedings of the Cancun Workshop on "Formation and evolution of massive young star clusters", eds: H.J.G.L.M. Lamers, A. Nota \& L.J. Smith.

Yusef-Zadeh, F., Cotton, W., Wardle, M., Melia, F. \& Roberts, D.A., 1994, ApJ, 434, L63.

Yusef-Zadeh, F., Morris, M, \& Chance, D., 1984, Nature, 310, 557.

Yusef-Zadeh, F. \& Morris, M., 1987, ApJ, 320, 545.

Yusef-Zadeh, F., Hewitt, J. W., \& Cotton, W., 2004, ApJSS, 155, 421-550.

Yusef-Zadeh, F., Wardle, M., \& Parastaran, P., 1997, ApJ, 475, L117.

Zeldovich, Ya. B., 1956, J. Exptl. Theoret. Phys. (U.S.S.R.), 31, 154.

Zweibel, E. G. \& Heiles, C., 1997, Nature, 385, 131. 